\title{
Preliminary evaluation of cone beam computed tomography in three-dimensional cephalometry for clinical application
}

\author{
NA $\mathrm{LI}^{1}, \mathrm{BO} \mathrm{HU}^{2}$, FANGLIN MI ${ }^{1}$ and JINLIN SONG ${ }^{2}$ \\ ${ }^{1}$ Department of Stomatology, Affiliated Hospital of North Sichuan Medical College, Nanchong, Sichuan 637000; ${ }^{2}$ Department \\ of Orthodontics, Stomatological Hospital Affiliated to Chongqing Medical University, Chongqing 401147, P.R. China
}

Received October 6, 2015; Accepted November 18, 2016

DOI: $10.3892 /$ etm.2017.4278

\begin{abstract}
The present study was conducted to evaluate the three-dimensional (3D) cephalometry accuracy of cone-beam computed tomography (CBCT). A total of 40 newly diagnosed orthodontic patients (including 18 males and 22 females; age range, 12-18 years) were subjected to CBCT scanning and $\mathrm{X}$-ray imaging in order to obtain lateral cephalograms. The 3D CBCT cephalograms were reconstructed and measured with in vivo 5.1 software, and compared with the results from the conventional 2D lateral cephalograms. Measurements in the two images were performed twice using the Steiner and Tweed standards and a single analyzer paired $t$-test was used to analyze the differences between the two cephalometric methods. The results indicated that the two methods showed significant differences in all 12 angle and 5 linear measurements $(\mathrm{P}<0.05)$. These findings indicated that $\mathrm{CBCT}$ is a more accurate technique compared with the conventional 2D method. In conclusion, CBCT may provide diagnostic and treatment information for maxillofacial deformities by using fast computer-aided analysis platform.
\end{abstract}

\section{Introduction}

Two-dimensional (2D) X-ray cephalometry for orthodontia was first used by Broadbent in 1931 (1), and this technique has since evolved from a manual to a computer-aided measurement $(2,3)$. Cephalometry is the foundation for the diagnosis, analysis and planning for orthodontic surgery and therapy. However, the 2D measurements based on radiation techniques are affected by a number of issues, such as image overlapping and landmark identification, achieved by positioning the patients body within a cone beam during CT scanning, thus

Correspondence to: Dr Jinlin Song, Department of Orthodontics, Stomatological Hospital Affiliated to Chongqing Medical University, 426 Songshibei Road, Yubei, Chongqing 401147, P.R. China

E-mail: songjinlin_sjl@sina.com

Key words: cone-beam computed tomography, orthodontics, cephalometry leading to low clarity of the lateral cephalograms for precision measurement (2-4).

With the advances (2-4) in computer software and medical technology, 3D cone-beam computed tomography (CBCT) for craniofacial cephalometry has become possible. This method can be applied to patient specific types of tissues with accurate results. 3D CBCT is easy to conduct. The technique has greatly improved and enhanced the breadth and depth of the applications of the 3D craniofacial structure imaging in clinical practices.

At present, there are several commonly used 3D cephalometric methods, including X-ray skull stereo imaging, 3D CT scanning and CBCT. CBCT is a relatively novel imaging method, with low radiation exposure of the patient, high scanning flexibility, image accuracy and fewer misdiagnosis, and is particularly suitable for hard tissue imaging $(5,6)$. These advantages have made CBCT increasingly popular in the diagnosis and treatment planning for various oral and craniofacial diseases $(7,8)$. However, due to the complexity of the 3D data, this method has not yet been standardized. Furthermore, the third-party software used in CBCT data analysis has not been adapted widely. Therefore, there is no single CBCT measurement method that has been widely used in clinical practices (9).

In the present study, CBCT-based cephalometry was attempted on craniofacial hard tissues with the specialized software in vivo, and the findings were compared the data obtained from the traditional 2D lateral radiographs.

\section{Materials and methods}

Patients and inclusion criteria. A total of 40 (including 18 males and 22 females, aged between 12 and 18 years) were randomly selected for inclusion into the present study from the Department of Orthodontics at the Stomatological Hospital Affiliated to Chongqing Medical University (Chongqing, China) between January 2009 and January 2011. Inclusion criteria were as follows: The patients had no missing teeth (with the exception of the third molar teeth), no history of trauma, no partial occlusion, no metal filling, no jaw or tooth tumors and no mandibular fracture in their permanent dentition. All patients have signed the consent statements and the study is approved by the Ethics committee of Stomatological Hospital Affiliated to Chongqing Medical University (Chongqing, China). 
Methods. All patients in the current study were subjected to X-ray and CBCT imaging. Traditional cephalograms were routinely obtained with an X-ray diagnosis system (Kodak 9000; Kodak, Rochester, NY, USA) at a voltage of $62 \mathrm{kV}$, current of $8 \mathrm{~mA}$ and distance of median sagittal plane to the X-ray source of $154.5 \mathrm{~cm}$. In addition, the amplification rate of the $\mathrm{X}$-ray imaging was $1.1 \mathrm{x}$ more than traditional cephalograms.

CBCT scans were conducted using the Classic i-CAT CBCT system (Imaging Science International, Hatfield, PA, USA) with the following parameters: Visible range was set at $13 \times 10 \mathrm{~cm}$ with gray value of $14 \mathrm{bit}$; scanning was performed at a speed of $360 \% \mathrm{sec}$ for $4 \mathrm{sec}$; the resolution was set to 0.4 stereo pixel at a layer thickness of $0.4 \mathrm{~mm}$; and the tube voltage and current were adjusted to $120 \mathrm{kV}$ and $5 \mathrm{~mA}$, respectively. During scanning, the patient was asked to sit in a centric occlusion position, using a chin pocket and a head frame to fix their head. The Frankfort plane was positioned parallel to the ground. The scanning baseline was aligned with the occlusal plane, and scanning was performed between the upper edges of the ears and the chin.

Marking and tracing of cephalometric landmarks using the Tweed and Steiner standards (10). The digitized X-ray cephalograms were uploaded into the WinCeph version 8.0 software (Rise Corporation, Sendai, Japan). The images were adjusted for brightness and contrast for better viewing. A total of 24 landmarks on the craniofacial hard tissues were marked and traced by an analyzer based on the scales marked on the X-ray films. These points were the following: Sella center, nasion, porion, basion O-orbital, pterygomaxillary fissure, anterior nasal spine posterior nasal spine, alveolar seat, lower alveolar seat, upper middle incisor, UlR-upper incisor teeth point, lower incisor, lower incisor root, upper molar, distinct upper molar, lower molar, gonion, pogonion, menton, gnathion center of mandibular bony joint, condyle vertex and posterior condylar.

CBCT scan data were fed to the DICOM 3.0 in vivo version 5.1 software (Anatomage, San Jose, CA, USA). The data were marked and traced by the same analyzer for the following 20 craniofacial hard tissue landmarks and profiles. Figs. 1 and 2 are representative three-dimensional images reconstructed from a cone-beam computed tomography scan using in vivo dental software and representative marked and traced image of three-dimensional cone-beam computed tomography scanning, obtained using in vivo dental software: Right orbital point, left orbital point, $\mathrm{N}$, right ear point, left ear point, right orbital profile, left orbital profile, right mandible profile, left mandible profile, symphyseal profile, S, Sella profile, nasion profile, Ba, upper right incisor profile, upper left incisor profile, lower right incisor profile, lower left incisor profile, maxillary profile and D.

Statistical analysis. Paired $t$-tests were conducted to determine differences between 17 measurements obtained from the 2D and 3D techniques using SPSS software (version 17.0; SPSS, Inc., Chicago, IL, USA). Differences were considered as statistically significant when $\mathrm{P}<0.05$. The data were also analyzed for the discrete degree indicators standard deviation (SD), standard error (SE) and coefficient of variation.

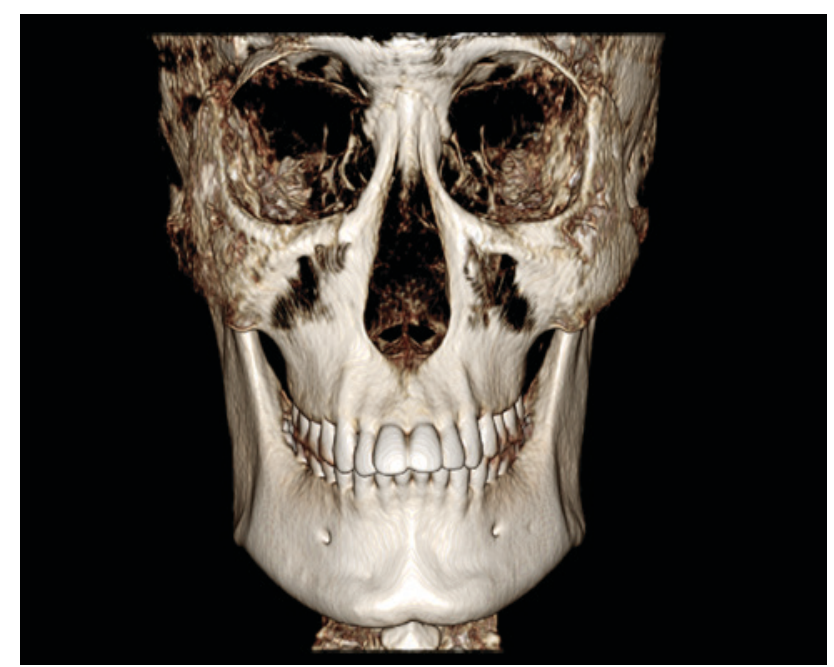

Figure 1. Representative three-dimensional image reconstructed from a cone-beam computed tomography scan using in vivo dental software.

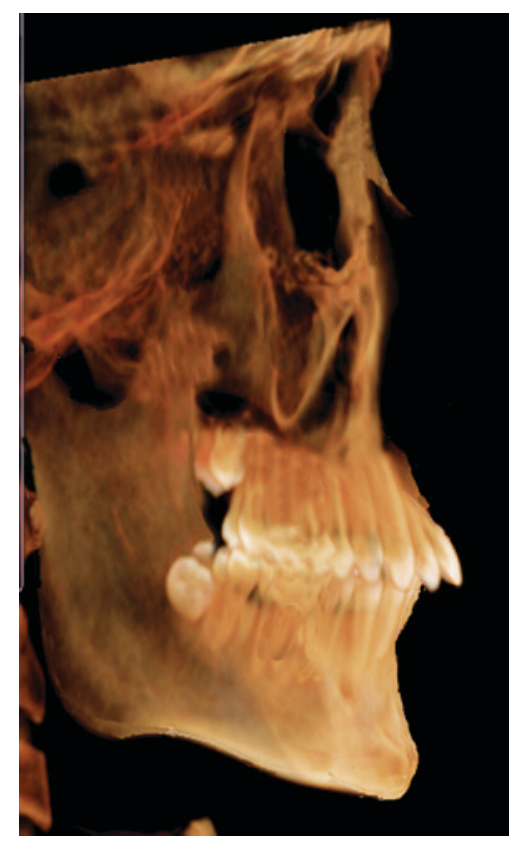

Figure 2. Representative marked and traced image of three-dimensional cone-beam computed tomography scanning, obtained using in vivo dental software.

\section{Results}

Cephalometric data from $2 D X$-ray images and $3 D C B C T$ cephalograms. Significant differences $(\mathrm{P}<0.05)$ were detected in all 12 angle and 5 linear measurements between the two methods (Table I). The discrete degree was higher in the 2D images than the 3D data (Fig. 1).

Comparison of the mean, SD, SE and coefficient of variation values between the two methods. Positioning of in vivo specimens is different from traditional multiplaner reconstruction and is based on four reference points at the N, Or-R, and left and right ears (Fig. 2). 3D measurements were less scattered and therefore, provided a higher degree of accuracy, compared with the 2D measurements, as determined by the standard 
Table I. Cephalometric data from 2D X-ray images and 3D CBCT cephalograms.

\begin{tabular}{|c|c|c|c|c|c|c|c|}
\hline \multirow[b]{2}{*}{ Measurement } & \multirow[b]{2}{*}{ Mean } & \multirow[b]{2}{*}{$\mathrm{SD}$} & \multirow[b]{2}{*}{ SE } & \multicolumn{2}{|c|}{$95 \% \mathrm{CI}$} & \multirow[b]{2}{*}{$t$-value } & \multirow[b]{2}{*}{ P-value } \\
\hline & & & & Minimum & Maximum & & \\
\hline SNA & 3.055 & 2.249 & 0.356 & 2.335 & 3.774 & 8.590 & $<0.001$ \\
\hline SNB & 1.578 & 1.870 & 0.296 & 0.979 & 2.176 & 5.336 & $<0.001$ \\
\hline ANB & 1.644 & 1.929 & 0.305 & 1.027 & 2.261 & 5.389 & $<0.001$ \\
\hline MP-FH & 4.758 & 4.471 & 0.707 & 3.328 & 6.188 & 6.730 & $<0.001$ \\
\hline SL & -3.013 & 7.843 & 1.240 & -5.521 & -0.504 & -2.429 & 0.002 \\
\hline SE & -1.114 & 1.713 & 0.271 & -1.662 & -0.566 & -4.114 & $<0.001$ \\
\hline SND & 3.300 & 2.149 & 0.340 & 2.613 & 3.988 & 9.711 & $<0.001$ \\
\hline U1-NA (mm) & 0.607 & 0.488 & 0.077 & 0.451 & 0.763 & 7.860 & $<0.001$ \\
\hline U1-NA $\left({ }^{\circ}\right)$ & -2.073 & 6.173 & 0.976 & -4.047 & -0.09865 & -2.124 & 0.040 \\
\hline U1-L1 & 4.489 & 9.074 & 1.435 & 1.587 & 7.391 & 3.129 & 0.003 \\
\hline L1-NB (mm) & -0.236 & 0.679 & 0.107 & -0.454 & -0.019 & -2.199 & 0.034 \\
\hline L1-NB $\left({ }^{\circ}\right)$ & 1.697 & 1.484 & 0.235 & 1.222 & 2.171 & 7.234 & $<0.001$ \\
\hline L1-MP & -3.437 & 5.205 & 0.823 & -5.102 & -1.772 & -4.176 & $<0.001$ \\
\hline L1-FH & -3.231 & 2.284 & 0.362 & -3.961 & -2.500 & -8.947 & $<0.001$ \\
\hline $\mathrm{OP}$ to $\mathrm{SN}$ & -1.801 & 1.479 & 0.234 & -2.274 & -1.327 & -7.699 & $<0.001$ \\
\hline Pog to NB (mm) & 0.298 & 0.844 & 0.134 & 0.028 & 0.568 & 2.232 & 0.031 \\
\hline GoGn to $\mathrm{SN}$ & 1.470 & 2.743 & 0.434 & 0.592 & 2.347 & 3.389 & 0.002 \\
\hline
\end{tabular}

CBCT, cone-beam computed tomography; SD, standard deviation; SE, standard error; CI, confidence interval.

Table II. Comparison of the mean, SD, SE and coefficient of variation values between the two methods.

\begin{tabular}{|c|c|c|c|c|c|c|}
\hline \multirow[b]{2}{*}{ Measurement } & \multicolumn{2}{|c|}{ Mean } & \multicolumn{2}{|c|}{$\mathrm{SD}$} & \multicolumn{2}{|c|}{ Coefficient of variation $(\%)$} \\
\hline & CBCT & Winceph8 & CBCT & Winceph8 & $\mathrm{CBCT}$ & Winceph 8 \\
\hline SNA & 84.88 & 87.32 & 1.576 & 1.941 & 1.86 & 2.22 \\
\hline SNB & 82.09 & 82.82 & 1.700 & 1.996 & 2.07 & 2.41 \\
\hline $\mathrm{ANB}$ & 2.93 & 2.90 & 1.115 & 1.124 & 38.08 & 38.76 \\
\hline MP-FH & 18.73 & 18.93 & 0.923 & 1.002 & 4.93 & 5.29 \\
\hline SL & 44.77 & 44.23 & 2.329 & 2.312 & 5.20 & 5.22 \\
\hline SE & 22.81 & 22.35 & 0.996 & 1.003 & 4.37 & 4.48 \\
\hline SND & 79.05 & 80.35 & 1.254 & 1.223 & 1.59 & 1.52 \\
\hline U1-NA (mm) & 6.49 & 6.32 & 0.362 & 0.367 & 5.58 & 5.80 \\
\hline U1-NA $\left({ }^{\circ}\right)$ & 40.73 & 39.02 & 1.129 & 1.171 & 2.77 & 3.00 \\
\hline U1-L1 & 102.89 & 101.39 & 1.072 & 1.078 & 1.04 & 1.06 \\
\hline L1-NB (mm) & 4.12 & 4.04 & 0.493 & 0.487 & 11.97 & 12.05 \\
\hline L1-NB $\left({ }^{\circ}\right)$ & 33.47 & 32.69 & 0.589 & 0.590 & 1.76 & 1.80 \\
\hline L1-MP & 108.31 & 107.02 & 1.760 & 1.775 & 1.63 & 1.66 \\
\hline L1-FH & 54.31 & 54.28 & 0.496 & 0.516 & 0.91 & 0.95 \\
\hline $\mathrm{OP}$ to $\mathrm{SN}$ & 20.90 & 20.20 & 0.762 & 0.792 & 3.65 & 3.92 \\
\hline Pog to NB (mm) & 1.83 & 1.77 & 0.476 & 0.484 & 2.60 & 2.73 \\
\hline GoGn to $\mathrm{SN}$ & 26.74 & 26.79 & 2.324 & 2.397 & 8.69 & 8.95 \\
\hline
\end{tabular}

SD, standard deviation; SE, standard error.

deviation and coefficient of variation values. Therefore, 3D CBCT may be able to provide more accurate data for orthodontic physicians for better therapeutic planning and have great potential clinical application (Table II). 


\section{Discussion}

Successful orthodontic treatment planning depends on accurate measurements on the cranio-maxillofacial skeleton, relative position of teeth, soft tissue lateral profile and craniofacial growth trends. The accuracy of such measurements is mainly affected by the quality of the lateral cephalograms (6).

Traditional 2D lateral cephalograms have certain inherent limitations. Firstly, it is difficult to precisely locate anatomical landmarks using $2 \mathrm{D}$ cephalograms due to image overlapping. Secondly, the images may present certain distortions as a result of different magnification due to differences in the distances between the film and craniofacial anatomy structures. Finally, 2D cephalogram measurements are easily affected by the head posture during imaging (9). Therefore, other techniques, such as 3D X-ray cephalography, 3D CT and 3D CBCT, have been developed to overcome these shortcomings. However, 3D X-ray cephalography is unable to present accurate cephalometric morphology for diagnosis and treatment (11-13). In addition, 3D CT imaging requires a long scanning time and higher radiation exposure for the patients. It also produces artifacts if there are metal fillings in the mouth and is therefore not recommended as a routine diagnosis method (14). CBCT is a computerized image reconstruction method developed from CT with an improved computational algorithm. Radiation exposure during this method is similar to that of X-ray cephalography $(15,16)$. Furthermore, cone-beam scanning improves the 3D spatial resolution, shortens the data acquisition time and reduces artifacts. CBCT has high quality imaging capacity and is easy to operate, thus may allow wide adaption by dentists in their clinical practices, and may contribute to accurate diagnosis and treatment of orthodontic diseases $(17,18)$.

Kumar et al (19) and van Vlijmen et al (20) observed that there was no significant measurement difference between different types of cephalograms. However, these studies used $2 \mathrm{D}$, not 3D, cephalograms for measurements. Following the comparison of 2D and 3D measurements, Gribel et al identified that there was no difference in linear measurements between 3D CBCT and direct dry skull measurements; however, a difference was detected between the 2D lateral X-ray imaging and direct dry skull measurement (21). Furthermore, Periago et al (22) and Baumgaertel et al (23) also demonstrated that 3D cephalometry was similar to direct skull measurement.

The results of the present study revealed that there were significant differences in all 12 angle and 5 linear measurements between the two methods (Tables I and II), and that 3D measurements were less scattered and therefore, a higher degree of accuracy, compared with the 2D measurements, as determined by the standard deviation and coefficient of variation values. These findings suggested that $3 \mathrm{D}$ CBCT has better measurement repeatability and accuracy when compared with the traditional 2D cephalometric measurements. Therefore, 3D CBCT may be able to provide more accurate data for orthodontic physicians for better therapeutic planning and have great potential clinical application.

In vivo 5.1 software (24) was used in the present study, which was developed by Anatomage specifically for CBCT image analysis. It can reconstruct 3D anatomical images from DICOM files generated by medical CT and magnetic resonance imaging instruments. The software can integrate the image, anatomical impression and CBCT data into a single file, which can then be opened by an application. Through the $3 \mathrm{D}$ analysis module, physicians create $3 \mathrm{D}$ cephalometric files that are compatible with the existing $2 \mathrm{D}$ analysis files. In addition, they are able to customize the modules by setting linear and point parameters, and to add new measurements (for example, other auxiliary points and lines, such as bisector of angle, perpendicular bisector and parallel line for better positioning and tracing). Additional programs can be coded to meet the requirements of different analyses and projects. All operations are easy, no additional device is required for the measurement, and all results are generated automatically and compared with the average from the patient specific group. The nasion is used as an origin to measure the linear distance at each angle.

In conclusion, the orthodontic in vivo application of aided CBCT technology remains at an early stage. The use of the $3 \mathrm{D}$ cephalometric method for orthodontic diagnosis is currently investigated further. The results of these investigations will provide theoretical support of the technology in orthodontic clinical practice and research. The current study demonstrated that the volumetric data obtained from a single CBCT scan can be processed by the image reconstruction techniques and used for cephalometric measurements to meet the clinical requirements prior to orthodontic surgery and examination for maxillofacial supernumerary teeth, embedded teeth and temporomandibular joint. This method can therefore be used to partially replace orthopantomogram and traditional cephalogram as the routine orthodontic examination option.

\section{Acknowledgements}

The present study was supported by the Research Projects, Department of Sichuan Education (Chengdu, China; grant no. 13ZB0251) and Nanchong Municipal Science and Technology Bureau (Sichuan, China; grant no. 13A0044).

\section{References}

1. Broadbent BH: A new x-ray technique and its application to orthodontia. Angle Orthod 1: 45-66, 1931.

2. Baumrind S and Frantz RC: The reliability of head film measurements. 1. Landmark identification. Am J Orthod 60: 111-127, 1971.

3. Li XZ, Wen XT, Zhou J and Wang P: X-ray film quality on hand-point repeatability influence. Zhongguo Xibu Kou Qiang Yi Xue Za Zhi 22: 342-343, 2004 (In Chinese).

4. Liu Y, Zhao J, Ding Y et al: Precision of cephalometric landmark identification from cone-beam computed tomography. Zhonghua Kou Qiang Yi Xue Za Zhi 17: 61-65, 2010 (In Chinese).

5. Feng Q and Qian Y: 3-Dimensional image measurements for craniofacial hard tissue. Kou Qiang Cai Liao Qi Xie Za Zhi 15: 140-142, 2006 (In Chinese).

6. Mah J and Hatcher D: Diagnóstico por imagen craneofacial enortodoncia. Capítulo 2. In: Grabber TM, Vanarsdall RL, VigKWL, eds. Orthodontics: Current Principles and Techniques. St Louis, MO: Elsevier; 71-100, 2005.

7. De Vos W, Casselman J and Swennen GR: Cone-beam computed tomography (CBCT) imaging of the oral and maxillofacial region: A systemic review of the literature. Int J Oral Maxillofac Surg 38: 609-625, 2009.

8. Arnheiter C, Scarfe WC and Farman AG: Trends in maxillofacial cone-beam computed tomography usage. Oral Radiology 22: 80-85, 2006. 
9. Major PW, Johnson DE, Hesse KL and Glover KE: Effect of head orientation on posterior anterior cephalometric landmark identification. Angle Orthod 66: 51-60, 1996.

10. Xü T, Ahn J and Baumrind S: Sensitivity of four representative angular cephalometric measures. Zhonghua Kou Qiang Yi Xue Za Zhi 35: 221-223, 2000 (In Chinese).

11. Baumrind S, Moffitt FH and Curry S: The geometry of three-dimensional measurement from paired coplanar x-ray images. Am J Orthod 84: 313-322, 1983.

12. Wang B, Li S and Zhou L: Development of a video system for three-dimensional cephalometry and dental cast analysis. Zhonghua Kou Qiang Yi Xue Za Zhi 35: 230-232, 2000 (In Chinese).

13. Yao S: Studies of three-dimensional measurement and analysis of cranio-mandibular-facial hard-soft tissue morphology and clinical application. PhD dissertation, The Fourth Military Medical School, Xi'an, 1993.

14. AYi-jiang, MiCong-bo. Application of 3D measurement techniques in orthodontics. Journal of Clinical Rehabilitative Tissue Engineering Research, 26 (15): 4869, 2011.

15. Swennen GR and Schutyser F: Three-dimensional cephalometry: Spiral multi-slice vs cone-beam computed tomography. Am J Orthod Dentofacial Orthop 130: 410-416, 2006.

16. Zhang JH, Chen WJ, Chen H and Fang Y: Clinical application of multi-slice spiral CT tooth surface imaging in the diagnosis of impacted tooth. Wen Zhou Yi Xue Yuan Xue Bao Bian Ji Bu 34: 221-222, 2004 (In Chinese).

17. Scarfe WC, Farman AG and Sukovic P: Clinical applications of cone-beam computed tomography in dental practice. J Can Dent Assoc 72: 75-80, 2006.
18. Zhang $\mathrm{J}$ and Zhu S: Advances in cone beam computed tomography technology for the quantitative measurements in orthodontics. Zhonghua Kou Qiang Yi Xue Za Zhi (electronic version) 4: 400-403, 2010 (In Chinese).

19. Kumar V, Ludlow J, Soares Cevidanes LH and Mol A: In vivo comparison of conventional and cone beam CT synthesized cephalograms. Angle Orthod 78:873-879, 2008.

20. van Vlijmen OJ, Bergé SJ, Swennen GR, Bronkhorst EM, Katsaros C and Kuijpers-Jagtman AM: Comparison of cephalometric radiographs obtained from cone-beam computed tomography scans and conventional radiographs. Int $\mathbf{J}$ Oral Maxillofac Surg 67: 92-97, 2009.

21. Gribel BF, Gribel MN, Frazäo DC, McNamara JA Jr and Manzi FR: Accuracy and reliability of craniometric measurements on lateral cephalometry and 3D measurements on CBCT scans. Angle Orthod 81: 26-35, 2011.

22. Periago DR, Scarfe WC, Moshiri M, Scheetz JP, Silveira AM and Farman AG: Linear accuracy and reliability of cone beam CT derived 3-dimensional images constructed using an orthodontic volumetric rendering program. Angle Orthod 78:387-395, 2008.

23. Baumgaertel S, Palomo JM, Palomo L and Hans MG: Reliability and accuracy of cone-beam computed tomography dental measurements. Am J Orthod Dentofacial Orthop 136: 19-28, 2009.

24. Ma X: Clinical Application of Maxillofacial Cone Beam CT. People's Health Publishing House, Beijing, pp94-995, 2011. 